\title{
Terahertz, Infrared, and UV-Vis Spectroscopy Study on Silver@Polyaniline Core@Shell Nanocomposites; Optical and Electronic Properties
}

\begin{abstract}
A. bouelsayed ${ }^{\dagger,} \neq$ Badawi Anis, ${ }^{*}, \dagger$ and Wael H Eisa ${ }^{\dagger}$
†Spectroscopy Department, Physics Division, National Research Centre, 33 El Bohouth Street (former El Tahrir Street), P.O. 12622, Dokki, Giza, Egypt.

キObour High Institute for Engineering and Technology, 31 KM Misr-Ismailia Road, Al Shorouk, Cairo, Egypt.
\end{abstract}

\section{Email: badawi.ali@daad-alumni.de}

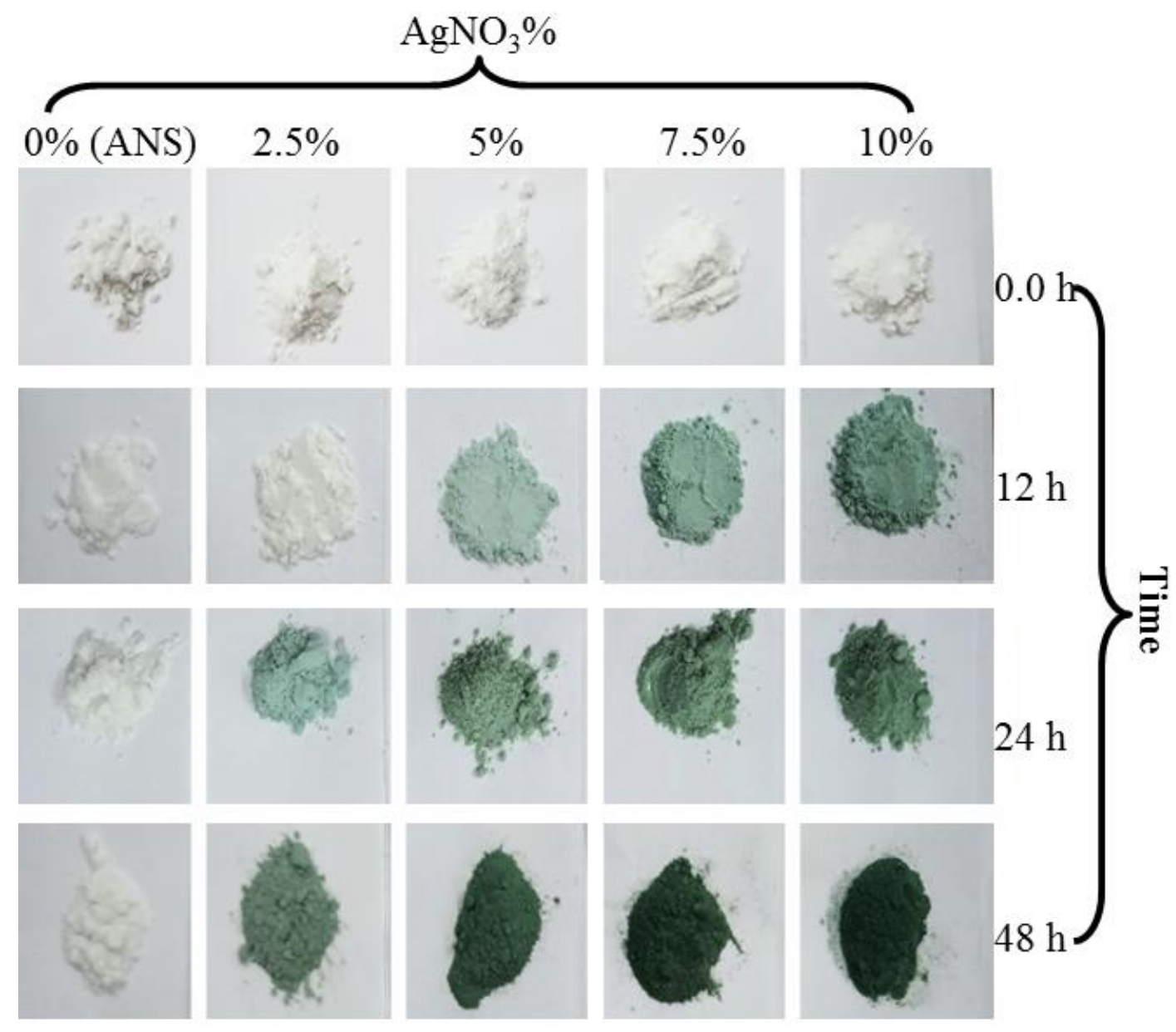

Figure S1: the complete color change of the ANS transformation to PANI from the beginning of mixing the reactants $\left(\mathrm{ANS}+\mathrm{AgNO}_{3}\right)$ till the end of the reaction after $48 \mathrm{~h}$. 


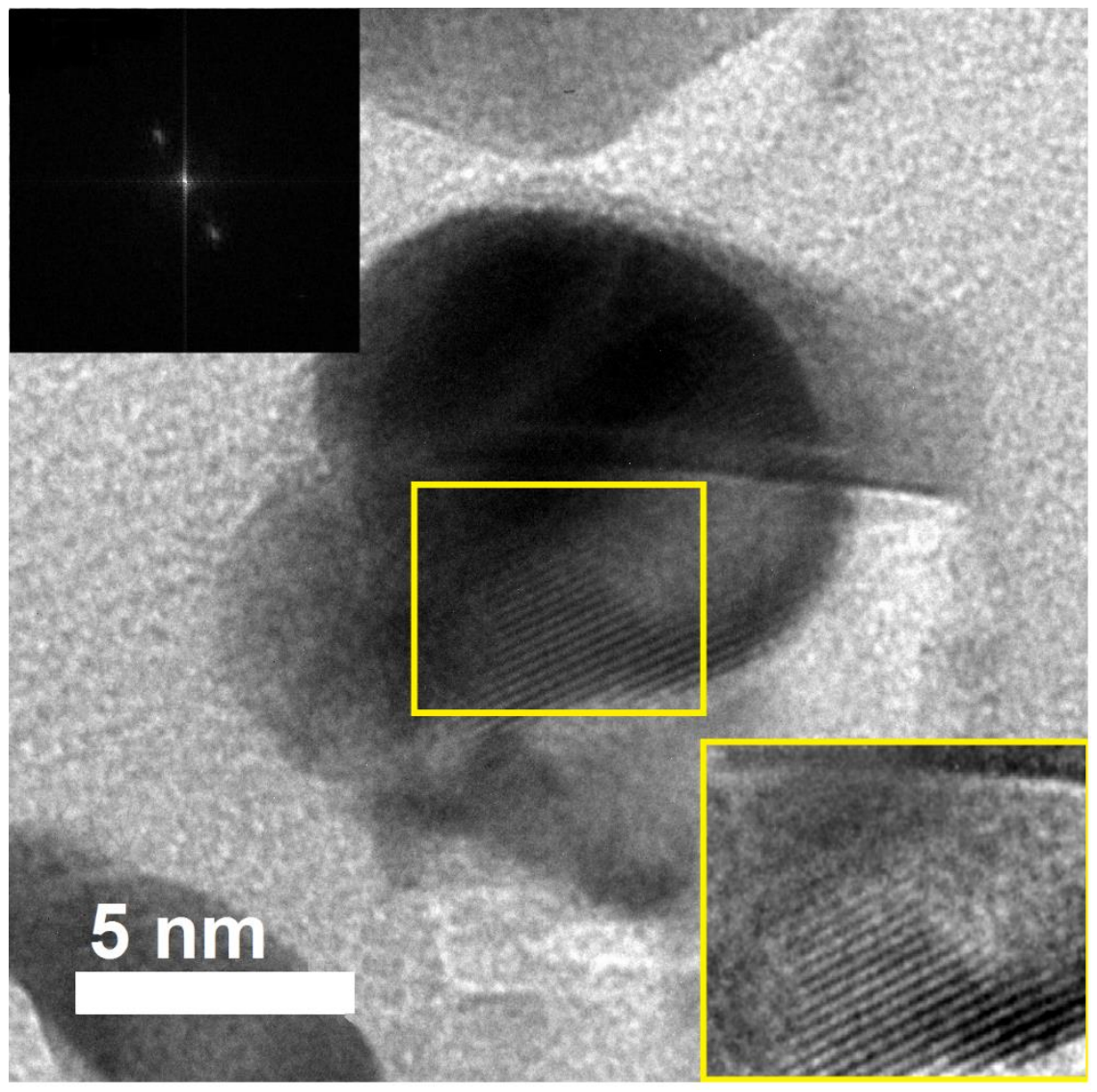

Figure S2: the image shows the presence of a higher contrast Ag core surrounded by a pale PANI core. The Ag regions displayed lattice fringes which are absent in PANI regions, indicating the existence of a crystalline Ag core and amorphous shell structure. The upper left inset shows the Fast Fourier Transform (FFT) and lower right inset is the fringes of d-spacing $\approx 21 \AA$ from the Ag (111) lattice spacing. 
Table S1: The elemental composition of ANS and 10 wt\% Ag@PANI core@ shell samples collected from the XPS survey spectra.

\begin{tabular}{ccc}
\hline \multirow{2}{*}{ Peak } & \multicolumn{2}{c}{ Atomic \% } \\
\cline { 2 - 3 } & ANS & 10wt\% Ag@ PANI \\
\hline C 1s & 45.8 & 72.06 \\
\hline Ag 3d & 33.53 & 1.48 \\
\hline O 1s & 8.83 & 12.73 \\
\hline N 1s & 11.84 & 10.92 \\
\hline S 2p & 45.8 & 2.81 \\
\hline
\end{tabular}

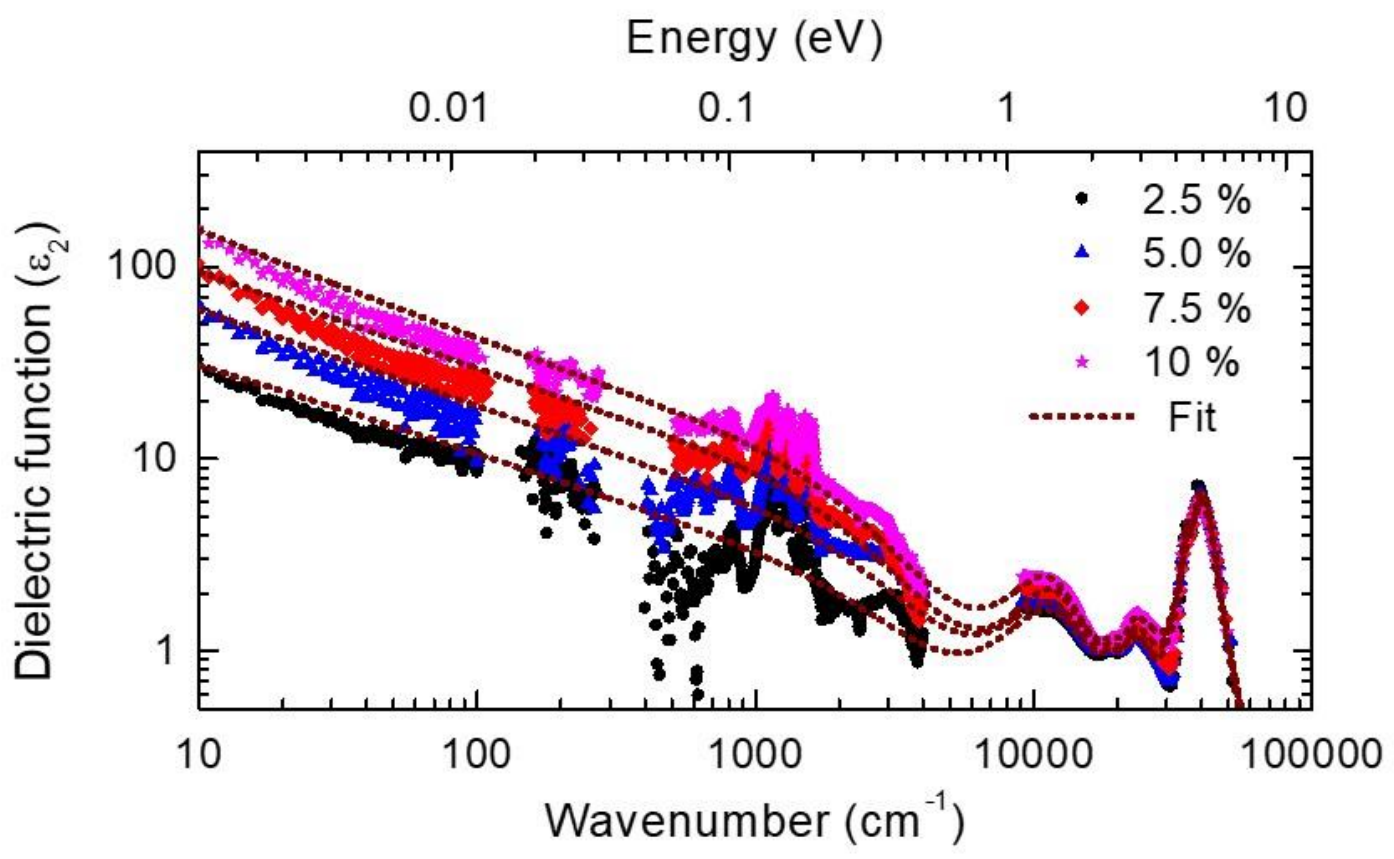

Figure S3: The values of the imaginary part $\varepsilon_{2}$ of the complex dielectric. The $\varepsilon_{2}$ values were used to calculate the real part of the optical conductivity through $\sigma_{\text {real }}=2 \omega \varepsilon_{0} \varepsilon_{2}$, where $\varepsilon_{0}=8.85 \times 10^{-12}$ $\mathrm{F} / \mathrm{m}$ is the permittivity of free space. 
Text S1 MATLAB code

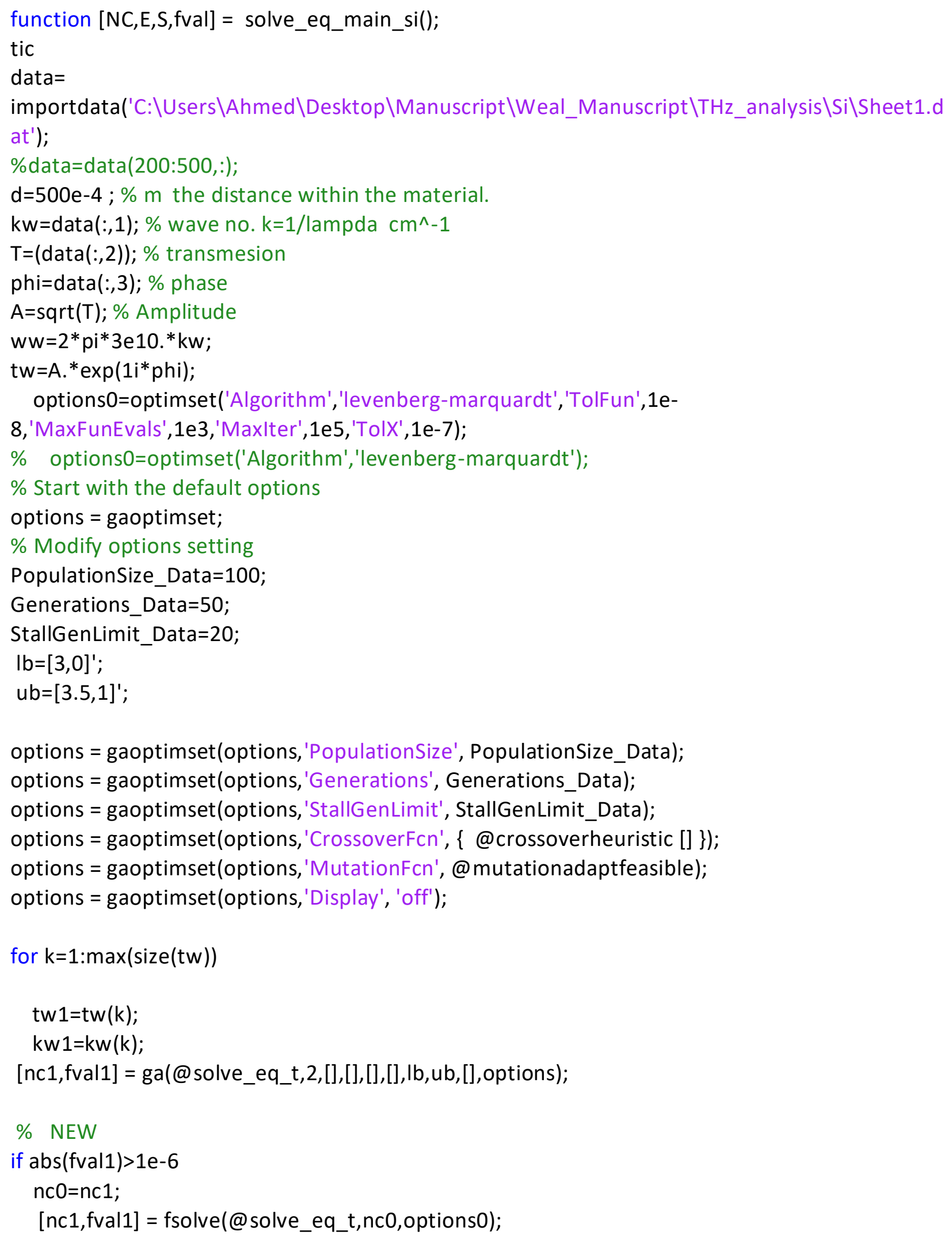


end

$\mathrm{NC}(\mathrm{k},:)=\mathrm{nc} 1$;

$\mathrm{fval}(k)=\mathrm{fval} 1$;

end

figure(1)

$\operatorname{plot}\left((\mathrm{kw}), \mathrm{NC}(:, 1),{ }^{\prime * \prime}\right)$;

figure(2)

$\operatorname{plot}\left((\mathrm{kw}), \mathrm{NC}(:, 2),{ }^{\prime * \prime}\right)$;

$n j=N C(:, 1)+1 i * N C(:, 2)$;

phid1=(nj). ${ }^{*} k w^{*} 2^{*}$ pi*d;

$\mathrm{TT}=\left(4 *(n j) .{ }^{*} \exp \left(1 i^{*}\right.\right.$ phid1)./((nj+1).^2-(nj-1).^2.*exp(1i*2*phid1)));

T_es=abs(TT).^2;

figure(3)

plot((kw),T,'r*',(kw),T_es, 'bo');

epsn=nj.^2;

$E=[$ real(epsn),imag(epsn)];

sig $=-i * 8.85 e-14 * 3 e 10 * 2 *$ pi*epsn.* $(k w)$;

$\mathrm{S}=[$ real(sig),imag(sig)];

$\max (f v a l)$, mean(fval)

toc

$\%$ Nested function that computes the objective function

function $F=$ solve_eq_t $(n c)$;

phid $=\left(n c(:, 1)+1 i^{*} n c(:, 2)\right) .{ }^{*} k w 1 * 2 * p i * d$;

$\mathrm{F}=\left(4^{*}\left(\mathrm{nc}(:, 1)+1 i^{*} \mathrm{nc}(:, 2)\right)\right.$. $^{*} \exp \left(1 \mathrm{i}^{*}\right.$ phid $) . /\left(\operatorname{eps}+\left(\left(\mathrm{nc}(:, 1)+1 \mathrm{i}^{*} \mathrm{nc}(:, 2)\right)+1\right) .^{\wedge} 2-\left(\left(\mathrm{nc}(:, 1)+1 \mathrm{i}^{*} \mathrm{nc}(:, 2)\right)-\right.\right.$

1).^2.*exp(1i*2*phid))-tw1);

$\% F=\left(4 *\left(n c(:, 1)+1 i^{*} n c(:, 2)\right) \cdot{ }^{*} \exp \left(1 i^{*} p h i d\right)-\operatorname{tw} 1 *^{*}\left(\left(\left(n c(:, 1)+1 i^{*} n c(:, 2)\right)+1\right) .^{\wedge} 2-\left(\left(n c(:, 1)+1 i^{*} n c(:, 2)\right)-\right.\right.\right.$

1).^2.*exp $\left(1 i^{*} 2 *\right.$ phid $\left.\left.)\right)\right)$;

$\mathrm{F}=\operatorname{sqrt}\left(\mathrm{F} \cdot{ }^{*} \operatorname{conj}(\mathrm{F})\right)$;

end

end 